\title{
JEAN-FRANÇOIS COLOMBEAU
}

\section{Quelques applications des fonctions implicites dans les espaces bornologiques}

Mémoires de la S. M. F., tome 31-32 (1972), p. 109-115

<http://www.numdam.org/item?id=MSMF_1972_31-32_109_0>

(C) Mémoires de la S. M. F., 1972, tous droits réservés.

L'accès aux archives de la revue « Mémoires de la S. M. F. » (http://smf. emath.fr/Publications/Memoires/Presentation.html) implique l'accord avec les conditions générales d'utilisation (http://www.numdam.org/conditions). Toute utilisation commerciale ou impression systématique est constitutive d'une infraction pénale. Toute copie ou impression de ce fichier doit contenir la présente mention de copyright.

\section{Numdam}


Colloque Anal. fonctionn.[1971, Bordeaux]

Bull. Soc. math. France,

Mémoire 31-32, 1972, p. 109-115.

QUELQUES APPLICATIONS DES FONCTIONS

IMPLICITES DANS LES ESPACES BORNOLOGIQUES

par

Jean François COLOMBEAU

Nous montrons comment on peut étendre les résultats obtenus par le théorème d'Ovcyannikov ([3] etc) à des situations plus générales et aussi "concrètes" grâce aux théorèmes des fonctions implicites du calcul différentiel dans les espaces bornologiques ([2] chap. XIII) en leur appliquant la méthode de majorations utilisée dans le théorème d'Ovcyannikov. Les notations et la terminologie sont conformes à[2]

$\S 1$ - Fonctions implicites dans les e.b.c. polaires complets.

THEOREME (1.1). - Soit $f$ continuement différentiable de $\mathrm{E}_{1} \times \mathrm{E}_{2}$ dans $\mathrm{E}_{3}$ et $(a, b) \in E_{1} \times E_{2}$ tel que $f(a, b)=0$ et $f_{y}^{\prime}(a, b) \in \operatorname{Isom}\left(E_{2}, E_{3}\right)$.

A) Existence : On suppose que pour tout $B_{1}$ disque borné de $E_{1}$ il existe $\tau_{0}>0$ et $C_{0}$ disque borné de $E_{2}$ fermé pour ${ }^{T E_{2}}$ tel que :

1) $f\left(a+\tau B_{1}, b\right) \subset \tau f_{y}^{\prime}(a, b) c_{0} \underline{s i} \tau \leqslant \tau$ et

2) qu'il existe une suite $\left(C_{n}\right)$ de disques bornés de $E_{2}$ fermés pour ${ }^{T E} E_{2}$ telle que :

$\left.\begin{array}{l}x \in a+\tau_{0} B_{1} \\ y \in b+\tau_{0}\left(c_{0}+C_{1}+\ldots+C_{n}\right)\end{array}\right\} \Rightarrow\left[f_{y}^{\prime}(x, y)-f_{y}^{\prime}(a, b)\right] C_{n} \subset f_{y}^{\prime}(a, b) C_{n+1}$

et telle que si $F_{n}=\bar{\Gamma}\left(\sum_{q \geqslant n} C_{q}\right) \quad F_{n} \rightarrow 0$ au sens de Mackey dans $E_{2}$ si $n \rightarrow+\infty$.

que :

Alors si $P_{1}=\bigcup_{B_{1}} \tau_{0} B_{1}$ il existe une application $u: a+P_{1} \rightarrow E_{2}$ telle

1) $u(a)=b$

2) $f(x, u(x))=0$ pour tout $x$ de $a+P_{I}$, et

3) $u$ est différentiable au point a et $u^{\prime}(a)=-f_{y}^{\prime}(a, b)^{-1} \circ f_{x}^{\prime}(a, b)$.

B) Unicité : On suppose que pour $B_{1}$ disque borné de $E_{1}$ et pour $C_{0}$ disque de $E_{2}$ fermé pour $T_{2}$ il existe une suite $\left(C_{n}\right)$ de disques de $E_{2}$ fermés pour $\mathrm{TE}_{2}$ telle que : 
$\left.\begin{array}{l}x \in a+B_{1} \\ y \in b+C_{0}\end{array}\right\} \Rightarrow\left[f_{y}^{\prime}(x, y)-f_{y}^{\prime}(a, b)\right] c_{n} \subset f_{y}^{\prime}(a, b) c_{n+1}$

et telle que $\bigcap_{n} C_{n}={ }^{\prime}\{0\}$.

Alors si $x \in a+B_{1}$ et si $y_{1}$ et $y_{2} \in b+C_{0}$

$f\left(x, y_{1}\right)=0=f\left(x, y_{2}\right) \Rightarrow y_{1}=y_{2}$.

Démonstration :

A) On suppose $a=b=0$; soit $T_{0}=f_{y}^{\prime}(0,0)$; soit $g: E_{1} \times E_{2} \rightarrow E_{2}$ définie par $g(x, y)=y-T_{0}^{-1} f(x, y)$; donc $f(x, y)=0 \Leftrightarrow y=g(x, y) \cdot g\left(x, y_{1}\right)-g\left(x, y_{2}\right)$ $=\mathrm{y}_{1}-\mathrm{y}_{2}-\mathrm{T}_{0}^{-1} \mathrm{f}\left(\mathrm{x}, \mathrm{y}_{1}\right)+\mathrm{T}_{0}^{-1} \mathrm{f}\left(\mathrm{x}, \mathrm{y}_{2}\right)=\mathrm{T}_{0}^{-1} \mathrm{~A}\left(\mathrm{x}, \mathrm{y}_{1}, \mathrm{y}_{2}\right)$ si $A\left(x, y_{1}, y_{2}\right)=f_{y}^{\prime}(0,0)\left(y_{1}-y_{2}\right)-f\left(x, y_{1}\right)+f\left(x, y_{2}\right)$, donc d'après le théorème des accroissements finis ([2] p. 142):

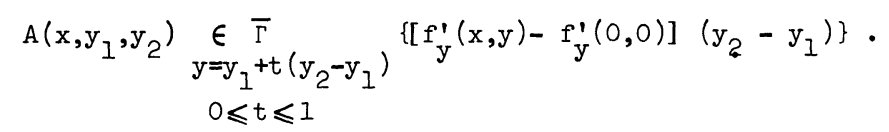

Soit $B_{1}$ un disque borné de $E_{1}$ et soit $x \in B_{1}$; soit $u_{0}(x)=0$, soit $u_{1}(x)=g\left(x, u_{0}(x)\right)=g(x, 0)$ donc $u_{1}(x)=-T_{0}^{-1} f(x, 0)$ donc si $0<\tau \leqslant \tau$ 。 $u_{1}\left(\tau B_{1}\right) \subset \tau C_{0}$; soit $u_{2}(x)=g\left(x, u_{1}(x)\right) ; u_{2}(x)-u_{1}(x)=g\left(x, u_{1}(x)\right)-g\left(x, u_{0}(x)\right)=$ $=T_{0}^{-1} A\left(x, u_{1}(x), u_{0}(x)\right) ; u_{1}(x) \in \tau C_{0}$ (si $x \in \tau B_{1}$ avec $\left.0<\tau \leqslant \tau_{0}\right)$ et $u_{0}(x)=0$ donc $u_{1}(x)-u_{0}(x) \in \tau C_{0}$ et donc d'après (I) et les hypothèses, $u_{2}(x)-u_{1}(x) \in \tau C_{1}$; on définit ainsi une suite $u_{n}(x)$ par $u_{n+1}(x)=g\left(x, u_{n}(x)\right)$; par récurrence on montre que $u_{n+1}(x)-u_{n}(x) \in \tau C_{n}$; donc comme la base de filtre $F_{n} \rightarrow 0$ au sens de Mackey si $n \rightarrow+\infty$, et comme $E_{2}$ est complet, si $P_{1}=\bigcup_{B_{1}} \tau_{0} B_{1}$ on dé finit une application $u: P_{1} \rightarrow E_{2}$ par $u(x)=\lim _{n \rightarrow+\infty} u_{n}(x) ; u_{n+1}(x) \in \tau\left(C_{0}+\ldots+C_{n}\right)$ si $x \in \tau B_{1}$ donc $u(x) \in \tau \bar{\Gamma}\left(\sum_{n \geqslant 0} C_{n}\right)$ donc $u$ est presque lipschitzienne au point 0 ; pour tout $n \quad u_{n}(0)=0$ donc $u(0)=0$; comme $f$ est $M$-continue dans $E_{1} \times E_{2}$ $g$ l'est aussi, donc par $M$-continuité $u(x)=g(x, u(x))$ pour tout $x \in P_{1}$ donc $f(x, u(x))=0$ pour tout $x \in P_{I}$. Donc d'après le lemme (1.2) (ci-dessous) u est différentiable au point 0 et $u^{\prime}(0)=-f_{y}^{\prime}(0,0)^{-1} \circ f_{x}^{\prime}(0,0)$.

B) Soient $x \in B_{1}, y_{1}$ et $y_{2} \in C_{0}$ tels que $f\left(x, y_{1}\right)=0=f\left(x, y_{2}\right)$; $\mathrm{y}_{1}-\mathrm{y}_{2}=\mathrm{g}\left(\mathrm{x}, \mathrm{y}_{1}\right)-\mathrm{g}\left(\mathrm{x}, \mathrm{y}_{2}\right)=\mathrm{T}_{0}^{-1} \mathrm{~A}\left(\mathrm{x}, \mathrm{y}_{1}, \mathrm{y}_{2}\right) \in 2 \mathrm{C}_{1}$ d'après ( $\mathrm{I}$ ) et les hypothèses car $y_{1}-y_{2} \in 2 C_{0}$; par récurrence, on montre que pour tout $n y_{1}-y_{2} \in 2 C_{n}$ donc $\mathrm{y}_{1}-\mathrm{y}_{2}=0$. 
LEMME (1.2) (Différentiabilité de la fonction implicite).

Soit $f$ continuement différentiable de $E_{1} \times E_{2}$ dans $E_{3}$ et $(a, b) \in E_{1} \times E_{2}$ tel que $f(a, b)=0$ et $f_{y}^{\prime}(a, b) \in \operatorname{Isom}\left(E_{2}, E_{3}\right)$. Soient $P_{1}$ une partie bornivore de $E_{1}$ et $u$ une application de ${ }^{a+P_{1}}$ dans $E_{2}$ telle que $u(a)=b$ et $f(x, u(x))=0$ pour tout $x \in a+P_{1} ;$ si $u$ est presque lipschitzienne au point $a$, alors $u$ est différentiable au point a et $u^{\prime}(a)=-f_{y}^{\prime}(a, b)^{-1} \circ f_{x}^{\prime}(a, b)$.

Démonstration : On suppose $a=b=0 ; f(h, k)-f(h, 0)-f_{y}^{\prime}(0,0) \cdot k \in \underset{0 \leqslant t \leqslant 1}{\bar{\Gamma}}$ $\left\{\left[f_{y}^{\prime}(h, t k)-f_{y}^{\prime}(0,0)\right] \cdot k\right.$ donc comme $f$ est continuement différentiable dans $U$ I'application $(h, k) \rightarrow f(h, k)-f(h, 0)-f_{y}^{\prime}(0,0) \cdot k$ est tangente à zéro; soient $S_{0}=f_{x}^{\prime}(0,0)$ et $T_{0}=f_{y}^{\prime}(0,0) ; f(h, u(h))=0$ si $h \in P_{I}$ donc $S_{0} h+T_{0} u(h)=-\left[f(h, u(h))-f(h, 0)-T_{0} u(h)\right]-\left[f(h, 0)-f(0,0)-S_{0} h\right] ;$ l'application $h \rightarrow f(h, 0)-f(0,0)-S_{0} h$ est tangente à zéro; comme u est presque lipschitzienne au point 0 , l'application $h \rightarrow f(h, u(h))-f(h, 0)-T_{0} u(h)$ est tangente à zéro donc l'application $h \rightarrow u(h)+T_{0}^{-1} S_{0} h$ est tangente à zéro, d'où le résultat.

Remarque (1.3): On montre par un exemple que dans le lemme (1.2) on ne peut pas en général remplacer " $u$ est presque lipschitzienne au point a " par " u est M-continue au point a" (comme on le fait dans le cas des espaces de Banach).

Remarque (1.4): Si $E_{1}, E_{2}$ et $E_{3}$ sont des espaces de Banach, le théorème (1.1) se réduit au théorème classique car les hypothèses techniques de (A) et (B) sont toujours vérifiées :

soient $B_{1}$ et $B_{2}$ les boules unités fermées de $E_{1}$ et $E_{2}$; $f$ étant continuement différentiable, il existe $\tau_{0}>0, \mu>0,0<k<1, \lambda \geqslant 0$ tels que

$$
\left.\begin{array}{l}
x \in a+\tau_{0} B_{1} \\
y \in b+\mu B_{2}
\end{array}\right\} \quad \Rightarrow f_{y}^{\prime}(a, b)^{-1}\left[f_{y}^{\prime}(x, y)-f_{y}^{\prime}(a, b)\right] B_{2} \subset k B_{2}
$$

et $f\left(a+\tau B_{1}, b\right) \subset \tau f_{y}^{\prime}(a, b)\left(\lambda B_{2}\right)$ si $0<\tau \leqslant \tau_{0}$; si on prend $\tau_{0}>0$ assez petit pour que $\frac{\tau_{0}{ }^{\lambda}}{1-k} \leqslant \mu$ on peut prendre $C_{n}=k^{n} \lambda B_{2}$ donc $F_{n} \subset \frac{k^{n} \lambda}{1-k} B_{2}$; de même, pour I'unicité.

Remarque (1.5) : Les hypothèses techniques du théorème (1.1) ne sont absolument pas superflues car on sait bien que le théorème des fonctions implicites tel qu'il 's'énonce dans les espaces de Banach devient faux dans le cadre des espaces de Fréchet ([1] p. 761). On peut évidemment énoncer des théorèmes un peu plus généraux que (1.1) ([2] p. 1.50). 


\section{$\S 2$ - Applications à des équations fonctionnelles.}

Le théorème des fonctions implicites dans les espaces de Banach permet notamment d'étudier des équations intégrales. Nous allons montrer sur un exemple très simple comment le théorème des fonctions implicites dans les espaces bornologiques permet d'étudier des équations intégro-différentielles, permettant ainsi" d'obtenir toute une gamme de résultats nouveaux en généralisant à des équations intégro-différentielles (notamment) les applications du théorème d'Ovcyannikov ou de théorèmes apparentés.

Soient $\mathrm{v}, \mathrm{a}, \mathrm{b}$ et $\mathrm{c}$ des fonctions analytiques définies au voisinage du point $\left(x_{0}, 0\right)$ de $\mathbb{\mathbb { L }}^{2}$ et à valeurs dans $\mathbb{\mathbb { N }}$. Soit $\mathrm{H}$ une fonction analytique définie au voisinage du point $\left(0,0, x_{0}\right)$ de $\mathbb{\mathbb { T }}^{3}$ et à valeurs dans $\mathbb{E}$. On cherche une fonction $u$ analytique au voisinage du point $\left(x_{0}, 0\right)$ de $\mathbb{\mathbb { L }}^{2}$, à valeurs dans $\mathbb{I}$ et satisfaisant l'équation :

$$
u(x, t)=v(x, t)+\int_{0}^{t} H(t, s, x)\left[a(x, s) \frac{\partial u}{\partial x}(x, s)+b(x, s) u(x, s)+c(x, s)\right] d s
$$

$\left(\int_{0}^{t}\right.$ signifie l'intégrale sur le segment $[0, t]=\underset{0 \leqslant \theta \leqslant 1}{\{\theta t\}} \underset{0}{0}$; les fonctions intégrées sont analytiques); cette équation contient l'équation aux dérivées partielles très simple considérée à titre d'exemple dans [2] p. 157.

$I_{\delta}$ désigne le disque ouvert de $\mathbb{L}$ défini par $|t|<\delta ; K$ désigne un compact de $\mathbb{I}$ dont l'intérieur contient le point $x_{0}^{(*)}$, si $s>0$ on définit $K+s={ }^{\prime}\{x \in \mathbb{I}$ tels que $d(x, K) \leqslant s\}$ (où $d(x, K)$ est la distance de $x$ au compact $K$ dans $R^{2}$ ); $K$ est choisi assez petit pour qu'il existe $s>0$ et $\delta>0$ tels que les fonctions $v, a, b$ et $c$ (respectivement $H$ ) sont définies dans un voisinage de $(\mathrm{K}+\mathrm{s}) \times \mathrm{I}_{\delta}$ (respectivement de $\mathrm{I}_{\delta}^{2} \times(\mathrm{K}+\mathrm{s})$ ).

On définit la bornologie de l'espace $\nLeftarrow(K)$ des germes de fonctions analytiques autour du compact $K$ par la base de la bomologie constituée (si $M$ et $s$ décrivent $10,+\infty]$ ) par les ' $\{\{M, s\}\}={ }^{\prime}\{\varphi$ analytiques à $I$ 'intérieur de $K+s$, continues sur $K+s$ et majorées par $M$ sur $K+s\}$ et $\&(K)$ est ainsi un ebc de Silva (donc polaire et complet).

Une partie $B$ de I'espace $C_{\infty}\left(I_{\delta}, H(K)\right.$ ) (voir [2] p. 146) est bornée si elle est constituée d'applications indéfiniment différentiables de $I_{\delta_{0}}$ dans $H(K)$ équibornées ainsi que leurs dérivées successives sur tout $I_{\delta}\left(0<\delta<\delta_{0}\right)$; mais la variable $t$ est complexe et donc d'après les formules intégrales de Cauchy (pour les applications analytiques à valeurs dans un ebc polaire) : pour qu'une partie $B$ de $C_{\infty}\left(I_{\delta_{0}}, \not(K)\right)$ soit bornée, il faut et il suffit qu'elle soit constituée d'applications indéfiniment différentiables de $I_{\delta}$ dans $\nLeftarrow(K)$ équibornées $\left(^{*}\right) K$ est supposé assez régulier pour que $\alpha\left(K+s, \mathfrak{C}\left(K+s^{+} s^{\prime}\right)\right)=s^{\prime}\left(s^{\prime} s, s^{\prime}\right.$ assez petits $)$. 
sur tout $I_{\delta}\left(0<\delta<\delta_{0}\right)$ (car il en est alors de même pour leurs dérivées successives). Donc une base de la bornologie de l'espace $C_{\infty}\left(I_{\delta_{0}}\right.$, $¥(K)$ ) est constituée (si

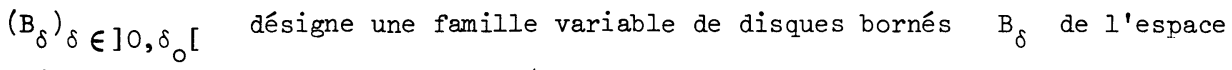
\& $(K))$ par les disques bornés $C=^{\prime}\left\{\xi \in C_{\infty}\left(I_{\delta_{0}}, \xi(K)\right)\right.$ tels que pour tout $\delta \in] 0, \delta_{0}\left[, t \in I_{\delta} \Rightarrow \xi(t) \in B_{\delta}\right\}$. Les disques bornés ' $\{\{M, s\}\}$ sont fermés dans $T H(K)$ et $\mathrm{si}$ les $\mathrm{B}_{\delta}$ sont fermés dans $T \&(K)$ le disque borné $C$ correspondant est fermé dans $T C_{\infty}\left(I_{\delta_{0}}, \xi(K)\right) \cdot C_{\infty}\left(I_{\delta_{0}}, \sharp(K)\right)$ est un ebc polaire complet ([2] p. 146).

Soit $K^{\prime}$ un compact de $\mathbb{C}^{2}$ contenant $(K+s) \times I_{\delta}$ (avec $\delta>\delta_{0}$ ) et tel que $\mathrm{v}$ soit analytique dans un voisinage de $\mathrm{K}^{\prime}$; soit $\mathrm{l}^{\prime}$ application $\mathrm{F}^{\prime}$ :

$\left[H\left(K^{\prime}\right) \times \mathbb{L}\right] \times C_{\infty}\left(I_{\delta_{0}}, H(K)\right) \rightarrow C_{\infty}\left(I_{\delta_{0}}, H(K)\right)$ définie par :

$$
\begin{aligned}
F(v, \lambda ; X)(t)(x)=X(t)(x)-v(x, t)-\lambda \int_{0}^{t} H(t, s, x) & {\left[a(x, s) \frac{d}{d x} X(s)(x)+\right.} \\
& +b(x, s) X(s)(x)+c(x, s)] d s .
\end{aligned}
$$

Il est immédiat de vérifier que $F$ est $C_{\infty} ; F(0,0 ; 0)=0$; $D_{2} F(0,0 ; 0)$ est I'application identique de $C_{\infty}\left(I_{\delta_{0}}\right.$, $\left.\nLeftarrow(K)\right)$ dans $C_{\infty}\left(I_{\delta_{0}}, \&(K)\right)$ et $D_{2} F(v, \lambda ; X) \cdot \xi(t)(x)=\xi(t)(x)-\lambda \int_{0}^{t} H(t, s, x)\left[a(x, s) \frac{d}{d x} \xi(s)(x)+b(x, s) \xi(s)(x)\right] d s$.

Nous allons appliquer le théorème des fonctions implicites à la fonction $F$ au point $(0,0)$ avec $E_{1}=H\left(K^{\prime}\right) \times \mathbb{L}$ et $E_{2}=E_{3}=C_{\infty}\left(I_{\delta_{0}}, z(K)\right)$. Nous allons vérifier le critère d'existence $(A)$.

$$
F(v, \lambda ; 0)(t)(x)=-v(x, t)-\lambda \int_{0}^{t} H(t, s, x) c(x, s) \text { ds ; soit } B_{1} \text { un disque borné }
$$
de $\sharp\left(K^{\prime}\right) \times \mathbb{L}$; il existe donc $M_{0}>0$ et $s_{0}>0$ tels que si $(v, \lambda) \in B_{1}$ nous pouvons prendre :

$$
\mathrm{C}_{0}=\left\{\xi \in C_{\infty}\left(I_{\delta_{0}}, H(K)\right) \text { tels que } \xi(t) \epsilon^{\prime}\left\{\left\{M_{0}, s_{0}\right\}\right\} \text { pour tout } t \in I_{\delta_{0}}\right\},
$$
(et si nous changeons de disque borné $B_{l}$ nous pouvons garder le même $s_{0}$ car $\left.K^{\prime} \supset(K+s) \times I_{\delta} \quad \begin{array}{ll}(\text { où } & s>0 \\ \text { et } & \delta>\delta_{0}\end{array}\right)$.

$$
\begin{array}{r}
{\left[D_{2} F(v, \lambda ; x)-D_{2} F(0,0 ; 0)\right](\xi)(t)(x)=-\lambda \int_{0}^{t} H(t, s, x)} \\
{\left[a(x, s) \frac{d}{d x} \xi(s)(x)+b(x, s) \xi(s)(x)\right] d s ;}
\end{array}
$$

$X$ et $v$ 'n'interviennent pas dans la formule $(I) ;(v, \lambda) \in B_{1}$ donc dans toute la

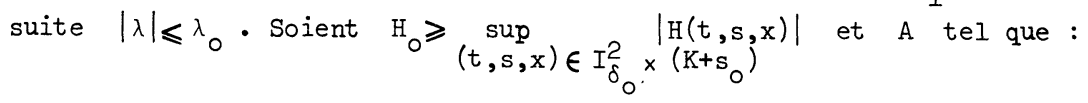




$$
\sup _{(x, t) \in\left(K+s_{0}\right) \times I_{\delta_{0}}}|a(x, t)|_{\frac{A}{2}} \quad \text { et } \quad \sup _{(x, t) \in\left(K+s_{0}\right)} \mid b(x, t) I_{\delta_{0}} \leqslant \frac{A}{2} .
$$

Pour la construction de la suite $\left(C_{n}\right)$ nous prenons :

$$
\begin{aligned}
& \mathrm{C}_{1} \supset \bar{\Gamma}_{|\lambda| \leqslant \lambda_{0}}\left[\mathrm{D}_{2} \mathrm{~F}(\mathrm{v}, \lambda ; \mathrm{X})-\mathrm{D}_{2} \mathrm{~F}(0,0 ; 0)\right] \mathrm{C}_{0} ; \xi \in \mathrm{C}_{0} \Rightarrow \mid \lambda \int_{0}^{t} \mathrm{H}(\mathrm{t}, \mathrm{s}, \mathrm{x}) \\
& {\left[a(x, s) \frac{d}{d x} \xi(s)(x)+b(x, s) \xi(s)(x)\right] d s k \lambda_{0} H_{0}|t|\left|\int_{0}^{1}\left(\frac{A}{2} \frac{M_{0}}{d}+\frac{A}{2} M_{0}\right) d \theta\right| \leqslant} \\
& \leqslant \frac{\lambda_{0} H_{0} A M_{0}|t|}{d} \text { si } x \in K+s_{0}-d \text { (pour d tel que } 0<d<\inf \left(1, s_{0}\right) \text { ) car } \\
& \varphi \in\left\{\left\{M_{0}, s_{0}\right\}\right\} \Rightarrow \frac{d \varphi}{d x} \in \epsilon^{\prime}\left\{\left\{\frac{M_{0}}{d}, s_{0}-d\right\}\right\} \text { (d'après la formule intégrale de Cauchy) } \\
& \text { et } M_{0} \leqslant \frac{M_{0}}{d} \text {. Donc si } B_{\delta}^{I}=\bigcap_{\substack{0<d<1 \\
s=s_{0}-d>0}}\left\{\left\{\frac{\lambda_{0} H_{0} A M_{0} \delta}{d}, s\right\}\right\}, C_{1} \subset\left\{\xi \in C_{\infty 0}\left(I_{\delta}, z(K)\right)\right. \\
& \text { tels que pour tout } \delta \in] 0, \delta_{0}\left[, t \in I_{\delta} \Rightarrow \xi(t) \in B_{\delta}^{I}\right\} \text {. }
\end{aligned}
$$

$$
\text { Nous allons montrer par récurrence sur } \mathrm{n} \text { que si : }
$$

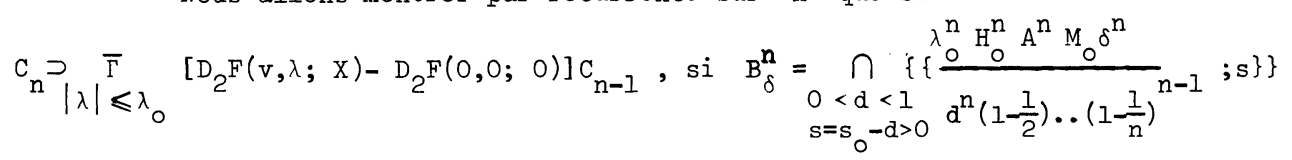

on peut prendre $C_{n}={ }^{\prime}\left\{\xi \in C_{\infty}\left(I_{\delta_{0}}, H(K)\right)\right.$ tels que pour tout $\left.\delta \in\right] 0, \delta_{0}[$, $\left.t \in I_{\delta} \Rightarrow \xi(t) \in B_{\delta}^{n}\right\}$.

Supposons donc $B_{\delta}^{n-1}=\bigcap_{\substack{0<d<1 \\ s=s_{0}-d>0}}\left\{\left\{\frac{\lambda_{0}^{n-1} H_{0}^{n-1} A^{n-1} M_{0} \delta^{n-1}}{d^{n-1}\left(1-\frac{1}{2}\right) \cdots\left(1-\frac{1}{n-1}\right)^{n-2}}, s\right\}\right\} ;$

changeons $d$ en $\left.d\left(1-\frac{1}{n}\right): B_{\delta}^{n-1} \subset \bigcap_{\substack{0<d<1 \\ s=s_{0}-d>0}} \cdot\left\{\frac{\lambda_{0}^{n-1} H_{0}^{n-1} A^{n-1} M_{0} \delta^{n-1}}{d^{n-1}\left(1-\frac{1}{2}\right) \ldots\left(1-\frac{1}{n}\right)^{n-1}}, s+\frac{d}{n}\right\}\right\}$

donc $\varphi \in B_{\delta}^{n-1} \Rightarrow \varphi$ et $\frac{d \varphi}{d x} \in\left\{\left\{\frac{\lambda_{0}^{n-1} H_{0}^{n-1} A^{n-1} M_{0} \delta^{n-1}}{d^{n}\left(1-\frac{1}{2}\right) \cdots\left(1-\frac{1}{n}\right)^{n-1}} \cdot n, s\right\}\right\}$

(d'après la formule intégrale de Cauchy).

$$
\begin{aligned}
& \text { Donc } \xi \in C_{n-1} \Rightarrow\left|\lambda \int_{0}^{t} H(t, s, x)\left[a(x, s) \frac{d}{d x} \xi(s)(x)+b(x, s) \xi(s)(x)\right] d s\right| \leqslant \\
& \leqslant \lambda_{0} H_{0}\left|\int_{0}^{1} A \frac{\lambda_{0}^{n-1} H_{0}^{n-1} A^{n-1} M_{0} \theta^{n-1}|t|^{n-1} n}{d^{n}\left(1-\frac{1}{2}\right) \ldots\left(1-\frac{1}{n}\right)^{n-1}}\right| t|d \theta| \leqslant \frac{\lambda_{0}^{n} H_{0}^{n} A^{n} M_{0}|t|^{n}}{d^{n}\left(1-\frac{1}{2}\right) \ldots\left(1-\frac{1}{n}\right)^{n-1}}
\end{aligned}
$$


d'où la formule annoncée pour $\mathrm{B}_{\delta}^{\mathrm{n}}$.

$$
F_{n}=\left(\bar{\Gamma} \sum_{q \geqslant n} C_{q}\right) \subset^{\prime}\left\{\xi \in C_{\infty}\left(I_{\delta_{0}}, \quad \forall(K)\right) \text { tels que pour tout } \delta \in\right] 0, \delta_{0}[
$$

$\left.t \in I_{\delta} \Rightarrow \xi(t) \in \bar{\Gamma} \sum_{q \geqslant n} B_{\delta}^{q}\right\} ;$ on sait que pour tout $n: \frac{1}{\left(1-\frac{1}{n}\right)^{n-1}}<e$ (e étant la base des logarithmes népériens), donc :

$$
B_{\delta}^{n} \subset \subset^{\prime \prime}\left\{\left\{\frac{M_{0}}{e}\left(\frac{\lambda_{0} H_{0} A \delta e}{d}\right)^{n}, s\right\}\right\}
$$

donc, si on fixe $d$ avec $0<d<1$ et $s=s_{0}-d>0$, pour $\delta_{0}$ assez petit la base de filtre $F_{n}$ converge vers 0 au sens de Mackey dans $C_{\infty}\left(I_{\delta_{0}}, \forall(K)\right.$ ) (et comme on $I^{\prime}$ a remarqué, si on change le disque borné de $H^{\prime}\left(K^{\prime}\right)$ en gardant $|\lambda| \leqslant \lambda_{0}$ on ne change pas ce $\delta_{0}$ car on ne change pas $s_{0}$, donc d) d'où : si $\delta_{0}>0$ est assez petit, il existe une application $\mathrm{U}: H\left(\mathrm{~K}^{\prime}\right) \rightarrow C_{\infty}\left(\mathrm{I}_{\delta_{0}}, H(\mathrm{~K})\right)$ telle que $\mathrm{U}(0)=0$ et pour tout $v \in\left\{\left(K^{\prime}\right) \quad F(v, l, U(v))=0\right.$; si on définit une fonction $u$ par $u(x, t)=U(t)(x), u$ est solution de l'équation (E) et $u$ est analytique en $x$ et $t$. On peut vérifier de manière analogue le critère d'unicité.

$\mathrm{Au}$ cours des calculs pour la construction de la suite des disques bornés $C_{n}$ nous avons utilisé une méthode qui est fondamentale dans la démonstration du théorème d'Ovcyannikov.

Il est bien évident que si on était parti d'une application plus "élaborée" du théorème d'Ovcyannikov on aurait pu de même la généraliser à des équations intégro-différentielles, obtenant ainsi des résultats nouveaux.

On peut aussi obtenir un théorème de Frobenius et lui appliquer la méthode de majoration d'Ovcyannikov et étudier ainsi des équations fonctionnelles nouvelles.

\section{BIBLIOGRAPHIE}

[1] EELLS (J.). - A setting for global analysis. Bull. Amer. Math. Soc. t. 72 (1966), p. 751-807.

[2] HOGBE-NLEND (H.). - Théorie des bornologies et applications. Berlin-Springer, Lecture Notes in Mathematics, Vol. 213.

[3] TREVES (F.). - Ovcyannikov theorem and hyperdifferential operators. IMPA, Ríio de Janeiro (1968).

Université de Bordeaux-I

U.E.R. Mathématiques et Informatique

351 cours de la Libération

33 - TALENCE (France) 